\title{
Maximum principles for hypersurfaces with vanishing curvature functions in an arbitrary Riemannian manifold
}

\author{
FRANCISCO X. FONTENELE ${ }^{1}$ and SÉRGIO L. SILVA ${ }^{2}$ \\ ${ }^{1}$ Universidade Federal Fluminense, Instituto de Matemática, Departamento de Geometria, \\ rua Mario Santos Braga s/no., 24020-140 Niterói, RJ, Brazil \\ ${ }^{2}$ Universidade Estadual do Rio de Janeiro-UERJ, Departamento de Estruturas Matemáticas-IME \\ 20550-013 RJ, Brazil
}

Manuscript received on October 30, 2001; accepted for publication on December 16, 2001; presented by MANFREDO DO CARMO

\begin{abstract}
In this paper we generalize and extend to any Riemannian manifold maximum principles for Euclidean hypersurfaces with vanishing curvature functions obtained by Hounie-Leite.
\end{abstract}

Key words: maximum principle, hypersurface, rth mean curvature.

\section{INTRODUCTION}

In this paper we generalize and extend to any Riemannian manifold maximum principles for hypersurfaces of the Euclidean space with vanishing curvature function, obtained by Hounie-Leite (1995 and 1999). In order to state our results, we need to introduce some notations and consider some facts. Given an hypersurface $M^{n}$ of a Riemannian manifold $N^{n+1}$, denote by $k_{1}(p), \ldots, k_{n}(p)$ the principal curvatures of $M^{n}$ at $p$ with respect to a unitary vector that is normal to $M^{n}$ at $p$. We always assume that $k_{1}(p) \leq k_{2}(p) \leq \cdots \leq k_{n}(p)$. The rth mean curvature $H_{r}(p)$ of $M^{n}$ at $p$ is defined by

$$
H_{r}(p)=\frac{1}{\left(\begin{array}{l}
n \\
r
\end{array}\right)} \sigma_{r}\left(k_{1}(p), \ldots, k_{n}(p)\right),
$$

where $\sigma_{r}: \mathbb{R}^{n} \rightarrow \mathbb{R}$ is the rth elementary symmetric function. It is easy to see that $\sigma_{r}$ is positive on the positive cone $\mathcal{O}^{n}=\left\{\left(x_{1}, \ldots, x_{n}\right) \in \mathbb{R}^{n}: x_{i}>0, \forall i\right\}$. Denote by $\Gamma_{r}$ the connected component 
of $\left\{\sigma_{r}>0\right\}$ that contains the vector $(1, \ldots, 1) \in \mathbb{R}^{n}$. It was proved in Gårding (1959) that $\Gamma_{r}$ is an open convex cone and that

$$
\Gamma_{1} \supset \Gamma_{2} \supset \cdots \supset \Gamma_{n}
$$

Moreover on $\Gamma_{r}, 1 \leq r \leq n$, it holds that (see Caffarelli et al. 1985, Proposition 1.1)

$$
\frac{\partial \sigma_{r}}{\partial x_{i}}>0, \quad 1 \leq i \leq n .
$$

As it was observed in Hounie-Leite (1995), the subset $\left\{\sigma_{r}=0\right\}$ can be decomposed as the union of $r$ continuous leaves $Z_{1}, \ldots, Z_{r}$, being $Z_{1}$ the boundary $\partial \Gamma_{r}$ of the cone $\Gamma_{r}$. Furthermore each leaf $Z_{j}$ may be identified with the graph of a continuous function defined in the plane $x_{1}+\cdots+x_{n}=0$. Following Hounie-Leite(1995), we say that a point $x=\left(x_{1}, \ldots, x_{n}\right) \in \mathbb{R}^{n}$ has rank $r$ if exactly $r$ components of $x$ do not vanish.

As in Fontenele-Silva (2001), given $p \in M^{n}$ and a unitary vector $\eta_{o}$ that is normal to $M^{n}$ at $p$, we can parameterize a neighborhood of $M^{n}$ containing $p$ and contained in a normal ball of $N^{n+1}$ as

$$
\varphi(x)=\exp _{p}\left(x+\mu(x) \eta_{o}\right)
$$

where the vector $x$ varies in a neighborhood $W$ of zero in $T_{p} M$ and $\mu: W \rightarrow \mathbb{R}$ satisfies $\mu(0)=0$ and $\nabla \mu(0)=0$, being $\nabla$ the gradient operator in the Euclidean space $T_{p} M$. Choosing a local orientation $\eta: W \rightarrow T_{\varphi(W)}^{\perp} M$ of $M^{n}$ with $\eta(0)=\eta_{o}$, we denote by $H_{r}(x)$ the rth mean curvature of $M^{n}$ at $\varphi(x)$ with respect to $\eta(x)$.

Given hypersurfaces $M$ and $M^{\prime}$ of $N^{n+1}$ that are tangent at $p$ and a unitary vector $\eta_{o}$ that is normal to $M$ at $p$, we parameterize $M$ and $M^{\prime}$ as in (4), obtaining correspondent functions $\mu: W \rightarrow \mathbb{R}$ and $\mu^{\prime}: W \rightarrow \mathbb{R}$, defined in a neighborhood $W$ of zero in $T_{p} M=T_{p} M^{\prime}$. As in Fontenele-Silva (2001), we say that $M$ remains above $M^{\prime}$ in a neighborhood of $p$ with respect to $\eta_{o}$ if $\mu(x) \geq \mu^{\prime}(x)$ for all $x$ in a neighborhood of zero. We say that $M$ remains on one side of $M^{\prime}$ in a neighborhood of $p$ if either $M$ is above $M^{\prime}$ or $M^{\prime}$ is above $M$ in a neighborhood of $p$. Finally, denote by $\vec{k}(p)=\left(k_{1}(p), \ldots, k_{n}(p)\right)$ and by $\overrightarrow{k^{\prime}}(p)=\left(k_{1}^{\prime}(p), \ldots, k_{n}^{\prime}(p)\right)$ the principal curvature vectors at $p$ of respectively $M$ and $M^{\prime}$.

We can now state our results:

THEOREM 1.a. Let $M$ and $M^{\prime}$ be hypersurfaces of $N^{n+1}$ that are tangent at $p$, with normal vectors pointing in the same direction. Suppose that $M$ remains on one side of $M^{\prime}$ and that $H_{r}(x)=H_{r}^{\prime}(x)$ in a neighborhood of zero in $T_{p} M$, for some $r, 1 \leq r<n$. If $r \geq 2$, suppose further that $\vec{k}(p)$ and $\overrightarrow{k^{\prime}}(p)$ belong to same leaf of $\left\{\sigma_{r}=0\right\}$ and the rank of either $\vec{k}(p)$ or $\overrightarrow{k^{\prime}}(p)$ is at least $r$. Then, $M$ and $M^{\prime}$ must coincide in a neighborhood of $p$.

THEOREM 1.b. Let $M$ and $M^{\prime}$ be hypersurfaces of $N^{n+1}$ with boundaries $\partial M$ and $\partial M^{\prime}$, respectively, and assume that $M$ and $M^{\prime}$, as well as $\partial M$ and $\partial M^{\prime}$, are tangent at $p \in \partial M \cap \partial M^{\prime}$, with normal 
vectors pointing in the same direction. Suppose that $M$ remains on one side of $M^{\prime}$ and that $H_{r}(x)=H_{r}^{\prime}(x)$ in a neighborhood of zero in $T_{p} M$, for some $r, 1 \leq r<n$. If $r \geq 2$, suppose further that $\vec{k}(p)$ and $\overrightarrow{k^{\prime}}(p)$ belong to same leaf of $\left\{\sigma_{r}=0\right\}$ and the rank of either $\vec{k}(p)$ or $\overrightarrow{k^{\prime}}(p)$ is at least $r$. Then, $M$ and $M^{\prime}$ must coincide in a neighborhood of $p$.

As a consequence of Theorems 1.a and 1.b, we obtain the following corollaries, that extend Theorem 0.1 in Hounie-Leite (1995) to any Riemannian manifold.

COROLlaRY 1.a. Let $M$ and $M^{\prime}$ be hypersurfaces of $N^{n+1}$ that are tangent at $p$, with normal vectors pointing in the same direction and with both having $r$-mean curvature equal to zero for some $r, 1 \leq r<n$. For $r \geq 2$, suppose further that $\vec{k}(p)$ and $\overrightarrow{k^{\prime}}(p)$ belong to same leaf of $\left\{\sigma_{r}=0\right\}$ and the rank of either $\vec{k}(p)$ or $\overrightarrow{k^{\prime}}(p)$ is at least $r$. Under these conditions, if $M$ remains on one side of $M^{\prime}$, then $M$ and $M^{\prime}$ must coincide in a neighborhood of $p$.

COROLlaRY 1.b. Let $M$ and $M^{\prime}$ be hypersurfaces of $N^{n+1}$ with boundaries $\partial M$ and $\partial M^{\prime}$, respectively, so that $M$ and $M^{\prime}$, as well as $\partial M$ and $\partial M^{\prime}$, are tangent at $p \in \partial M \cap \partial M^{\prime}$, with normal vectors pointing in the same direction. Assume that $M$ and $M^{\prime}$ have r-mean curvature equal to zero for some $r, 1 \leq r<n$. For $r \geq 2$, suppose further that $\vec{k}(p)$ and $\overrightarrow{k^{\prime}}(p)$ belong to same leaf of $\left\{\sigma_{r}=0\right\}$ and the rank of either $\vec{k}(p)$ or $\overrightarrow{k^{\prime}}(p)$ is at least $r$. Under these conditions, if $M$ remains on one side of $M^{\prime}$, then $M$ and $M^{\prime}$ must coincide in a neighborhood of $p$.

The extension of Theorem 1.3 in Hounie-Leite (1999) is given in the following theorems.

THEOREM 2.a. Let $M$ and $M^{\prime}$ be hypersurfaces of $N^{n+1}$ that are tangent at $p$, with normal vectors pointing in the same direction. Suppose that $M$ remains above $M^{\prime}$ and that $H_{r}^{\prime} \geq 0 \geq H_{r}$, for some $r, 2 \leq r<n$. Suppose further that $H_{j}^{\prime}(p) \geq 0,1 \leq j \leq r-1$, and either $H_{r+1}(p) \neq 0$ or $H_{r+1}^{\prime}(p) \neq 0$. Then, $M$ and $M^{\prime}$ must coincide in a neighborhood of $p$.

THEOREM 2.b. Let $M$ and $M^{\prime}$ be hypersurfaces of $N^{n+1}$ with boundaries $\partial M$ and $\partial M^{\prime}$, respectively, and assume that $M$ and $M^{\prime}$, as well as $\partial M$ and $\partial M^{\prime}$, are tangent at $p \in \partial M \cap \partial M^{\prime}$ with normal vectors pointing in the same direction. Suppose that $M$ remains above $M^{\prime}$ and that $H_{r}^{\prime} \geq 0 \geq H_{r}$, for some $r, 2 \leq r<n$. Suppose further that $H_{j}^{\prime}(p) \geq 0,1 \leq j \leq r-1$, and either $H_{r+1}(p) \neq 0$ or $H_{r+1}^{\prime}(p) \neq 0$. Then $M$ and $M^{\prime}$ must coincide in a neighborhood of $p$.

It will be clear from the proofs that in Theorems 2 a and 2.b we only need to require $H_{r}^{\prime}(x) \geq$ $H_{r}(x)$, in a neighborhood of zero in $T_{p} M$, and $H_{r}^{\prime}(p) \geq 0 \geq H_{r}(p)$ instead of $H_{r}^{\prime} \geq 0 \geq H_{r}$ everywhere. For $r=1$, it must be observed that, in Theorems 2.a and 2.b, we can assume only that $H_{r}^{\prime}(x) \geq H_{r}(x)$ and that $M$ remains above $M^{\prime}$ in a neighborhood of zero in $T_{p} M$ (see Theorems 1.1 and 1.2 in Fontenele-Silva (2001)).

\section{PRELIMINARIES}

In this section we will present the necessary material for our proofs.

Following Hounie-Leite (1995), we say that $x \in \mathbb{R}^{n}$ is an elliptic root of $\sigma_{r}$ if $\sigma_{r}(x)=0$ and either $\frac{\partial \sigma_{r}}{\partial x_{j}}(x)>0, j=1, \ldots, n$, or $\frac{\partial \sigma_{r}}{\partial x_{j}}(x)<0, j=1, \ldots, n$. It is easy to see that any root of 
$\sigma_{1}=0$ is elliptic. For $2 \leq r<n$, we have the following criterion of ellipticity (see Corollary 2.3 in Hounie-Leite (1995) and Lemma 1.1 in Hounie-Leite (1999)):

Lemma 1. Let $x \in \mathbb{R}^{n}$ and assume that $\sigma_{r}(x)=0$ for some $2 \leq r<n$. Then, the following conditions are equivalent

(i) $x$ is elliptic.

(ii) the rank of $x$ is at least $r$.

(iii) $\sigma_{r+1}(x) \neq 0$.

For the proofs of our results, we will also need of the following lemmas:

Lemma 2. If $y, w$ belong to a leaf $Z_{j}$ of $\sigma_{r}=0, w-y$ belongs to the closure $\overline{\mathcal{O}^{n}}$ of $\mathcal{O}^{n}$ and either $y$ or $w$ is an elliptic root, then $y=w$.

Lemma 3. For $1 \leq r \leq n$, if $x \in \mathbb{R}^{n}$ satisfies $\sigma_{j}(x) \geq 0,1 \leq j \leq r$, then $x \in \overline{\Gamma_{r}}$.

Lemma 2 is a particular case of Lemma 1.3 in Hounie-Leite (1995) and Lemma 3 follows from the proof of Lemma 1.2 in Hounie-Leite (1999).

For $d=(n(n+1) / 2)+2 n+1$, write an arbitrary point $p$ at $\mathbb{R}^{d}$ as

$$
p=\left(r_{11}, \ldots, r_{1 n}, r_{22}, \ldots r_{2 n}, \ldots, r_{(n-1) n}, r_{n n}, r_{1}, \ldots, r_{n}, z, x_{1}, \ldots, x_{n}\right)
$$

or, in short, as $p=\left(r_{i j}, r_{i}, z, x\right)$ with $1 \leq i \leq j \leq n$, and $x=\left(x_{1}, \ldots, x_{n}\right)$. A $C^{1}$-function $\Phi: \Gamma \rightarrow \mathbb{R}$ defined in an open set $\Gamma$ of $\mathbb{R}^{d}$ is said to be elliptic in $p \in \Gamma$ if

$$
\sum_{i \leq j=1}^{n} \frac{\partial \Phi}{\partial r_{i j}}(p) \xi_{i} \xi_{j}>0 \text { for all nonzero }\left(\xi_{1}, \xi_{2}, \ldots, \xi_{n}\right) \in \mathbb{R}^{n}
$$

We say that $\Phi$ is elliptic in $\Gamma$ if $\Phi$ is elliptic in $p$ for all $p \in \Gamma$. Given a function $f: U \rightarrow \mathbb{R}$ of class $C^{2}$, defined in an open set $U \subset \mathbb{R}^{n}$, and $x \in U$, we associate a point $\Lambda(f)(x)$ in $\mathbb{R}^{d}$ setting

$$
\Lambda(f)(x)=\left(f_{i j}(x), f_{i}(x), f(x), x\right)
$$

where $f_{i j}(x)$ and $f_{i}(x)$ stand for $\frac{\partial^{2} f}{\partial x_{i} \partial x_{j}}(x)$ and $\frac{\partial f}{\partial x_{i}}(x)$, respectively. Saying that the function $\Phi$ is elliptic with respect to $f$ means that $\Lambda(f)(x)$ belongs to $\Gamma$ and $\Phi$ is elliptic in $\Lambda(f)(x)$ for all $x \in U$. For elliptic functions it holds the following maximum principle(see Alexandrov 1962):

MaXimum Principle. Let $f, g: U \rightarrow \mathbb{R}$ be $C^{2}$-functions defined in an open set $U$ of $\mathbb{R}^{n}$ and let $\Phi: \Gamma \subset \mathbb{R}^{d} \rightarrow \mathbb{R}$ be a function of class $C^{1}$. Suppose that $\Phi$ is elliptic with respect to the functions $(1-t) f+t g, t \in[0,1]$. Assume also that

$$
\Phi(\Lambda(f)(x)) \geq \Phi(\Lambda(g)(x)) \quad, \forall x \in U
$$


and that $f \leq g$ on $U$. Then, $f<g$ on $U$ unless $f$ and $g$ coincide in a neighborhood of any point $x_{o} \in U$ such that $f\left(x_{o}\right)=g\left(x_{o}\right)$.

Consider now a hypersurface $M^{n} \subset N^{n+1}$, a point $p \in M$ and a unitary vector $\eta_{o}$ that is normal to $M^{n}$ at $p$. Fix an orthonormal basis $e_{1}, \ldots, e_{n}$ in $T_{p} M$ and introduce coordinates in $T_{p} M$ by setting $x=\sum_{i=1}^{n} x_{i} e_{i}$ for all $x \in T_{p} M$. Parameterize a neighborhood of $p$ in $M$ as in (4), obtaining a function $\mu: W \subset T_{p} M \rightarrow \mathbb{R}$. Recall that $\mu(0)=0$ and $\frac{\partial \mu}{\partial x_{i}}(0)=0$, for all $i, 1 \leq i \leq n$. Choose a local orientation $\eta: W \rightarrow T_{\varphi(W)}^{\perp} M$ of $M^{n}$ with $\eta(0)=\eta_{o}$ and denote by $A_{\eta(x)}$ the second fundamental form of $M^{n}$ in the direction $\eta(x)$. Denote by $\varphi_{i}(x)$ the vector $\frac{\partial \varphi}{\partial x_{i}}(x)$ and by $A(x)=\left(a_{i j}(x)\right)$ the matrix of $A_{\eta(x)}$ in the basis $\varphi_{i}(x)$. In Fontenele-Silva (2001), it is proved the existence of an $n \times n$-matrix valued function $\tilde{A}$ defined in an open set $\mathbb{R}^{(n(n+1) / 2)+n} \times \mathcal{N} \subset \mathbb{R}^{d}$, being $\mathcal{N}$ an open set of $\mathbb{R}^{n+1}$, containing the origin of $\mathbb{R}^{d}$ such that

$$
\tilde{A}(\Lambda(\mu)(x))=A(x), \quad x \in W .
$$

Moreover, we have $\tilde{A}\left(r_{i j}, r_{i}, z, x\right)$ diagonalizable for all $\left(r_{i j}, r_{i}, z, x\right) \in \mathbb{R}^{(n(n+1) / 2)+n} \times \mathcal{N}$. Consider the function $\Phi_{r}: \mathbb{R}^{(n(n+1) / 2)+n} \times \mathcal{N} \rightarrow \mathbb{R}$ defined by

$$
\Phi_{r}=\frac{1}{\left(\begin{array}{l}
n \\
r
\end{array}\right)} \sigma_{r} \circ \lambda \circ \tilde{A},
$$

where $\lambda(\tilde{A}(w))=\left(\lambda_{1}(\tilde{A}(w)), \ldots, \lambda_{n}(\tilde{A}(w))\right.$ for all $w \in \mathbb{R}^{(n(n+1) / 2)+n} \times \mathcal{N}$. Here $\lambda_{1}(\tilde{A}(w)) \leq$ $\cdots \leq \lambda_{n}(\tilde{A}(w))$ are the eigenvalues of $\tilde{A}(w)$. It follows from (1), (8) and (9) that

$$
H_{r}(x)=\Phi_{r}(\Lambda(\mu)(x)), x \in W
$$

The proof of Proposition 3.4 in Fontenele-Silva (2001) gives

$$
\sum_{k \leq \ell=1}^{n} \frac{\partial \Phi_{r}}{\partial r_{k \ell}}\left(r_{i j}, 0,0,0\right) \xi_{k} \xi_{\ell}=\frac{1}{\left(\begin{array}{l}
n \\
r
\end{array}\right)} \sum_{k, \ell=1}^{n} \frac{\partial\left(\sigma_{r} \circ \lambda\right)}{\partial A_{k \ell}}\left(\tilde{A}\left(\left(r_{i j}, 0,0,0\right)\right)\right) \xi_{k} \xi_{\ell},
$$

for all $\left(r_{i j}, 0,0,0\right) \in \mathbb{R}^{d}$.

We also make use of the following lemma

Lemma 4. If $A_{o} \in \mathcal{M}^{n}(\mathbb{R})$ is symmetric and $\frac{\partial \sigma_{r}}{\partial \lambda_{i}}\left(\lambda\left(A_{o}\right)\right)>0 \quad(<0)$ for all $1 \leq i \leq n$, then

$$
\sum_{i, j=1}^{n} \frac{\partial\left(\sigma_{r} \circ \lambda\right)}{\partial A_{i j}}\left(A_{o}\right) \xi_{i} \xi_{j}>0 \quad(<0), \quad \forall \xi=\left(\xi_{1}, \ldots, \xi_{n}\right) \neq 0 .
$$

The proof of Lemma 4 follows from the proof of Lemma 3.3 in Fontenele-Silva (2001).

\section{PROOFS OF OUR RESULTS}

We will prove only Theorems 1.a and 2.a, since the proofs of Theorems 1.b and 2.b are analogous. 
Proof of Theorem 1.a. If $r=1$, the theorem follows from Theorem 1.1 in Fontenele-Silva (2001). Thus, we assume that $2 \leq r<n$. The assumption $H_{r}(x)=H_{r}^{\prime}(x)$ in a neighborhood $W$ of zero in $T_{p} M$ and (10) imply that

$$
\Phi_{r}(\Lambda(\mu)(x))=\Phi_{r}\left(\Lambda\left(\mu^{\prime}\right)(x)\right), \quad x \in W
$$

On the other hand, $\vec{k}(p)$ and $\overrightarrow{k^{\prime}}(p)$ are both roots of $\sigma_{r}=0$ and one of them is elliptic by our hypothesis and Lemma 1 . The fact that $M$ remains on one side of $M^{\prime}$ implies that either $\vec{k}(p)-\overrightarrow{k^{\prime}}(p)$ or $\overrightarrow{k^{\prime}}(p)-\vec{k}(p)$ belongs to $\overline{\mathcal{O}^{n}}$. Since $\vec{k}(p)$ and $\overrightarrow{k^{\prime}}(p)$ belong to same leaf of $\left\{\sigma_{r}=0\right\}$ by assumption, it follows from Lemma 2 that

$$
\vec{k}(p)=\overrightarrow{k^{\prime}}(p)
$$

For each $t \in[0,1]$, if we consider the hypersurface $M_{t}$ parameterized by

$$
\varphi(x)=\exp _{p}\left(x+\left((1-t) \mu+t \mu^{\prime}\right)(x) \eta_{o}\right), \quad x \in W,
$$

we have that $M_{t}$ is tangent to both $M$ and $M^{\prime}$ in $p$ and that $M_{t}$ is between $M$ and $M^{\prime}$ in a neighborhood of $p$. Using (14), we conclude that the principal curvature vector of $M_{t}$ at $p$ is equal to $\vec{k}(p)=\overrightarrow{k^{\prime}}(p)$, for all $t \in[0,1]$. This implies, by (8), that

$$
\lambda \circ \tilde{A}\left((1-t) \Lambda(\mu)(0)+t \Lambda\left(\mu^{\prime}\right)(0)\right)=\vec{k}(p)=\overrightarrow{k^{\prime}}(p),
$$

for all $t \in[0,1]$. Since $\vec{k}(p)=\overrightarrow{k^{\prime}}(p)$ is elliptic, it follows from (11) and Lemma 4 that either $\Phi_{r}$ or $-\Phi_{r}$ is elliptic along the line segment $(1-t) \Lambda(\mu)(0)+t \Lambda\left(\mu^{\prime}\right)(0) \subset \mathbb{R}^{(n(n+1) / 2)+n} \times \mathcal{N} \subset \mathbb{R}^{d}$. Since ellipticity is an open condition, restricting $W$ if necessary, we conclude by continuity and by the compactness of $[0,1]$ that either $\Phi_{r}$ or $-\Phi_{r}$ is elliptic in $(1-t) \Lambda(\mu)(x)+t \Lambda\left(\mu^{\prime}\right)(x)$, for all $t \in[0,1]$ and $x \in W$. Consequently either $\Phi_{r}$ or $-\Phi_{r}$ is elliptic with respect to the functions $(1-t) \mu+t \mu^{\prime}, t \in[0,1]$. So, by (13), we can apply the maximum principle to conclude that $\mu$ and $\mu^{\prime}$ coincide in a neighborhood of zero. Therefore, $M$ and $M^{\prime}$ coincide in a neighborhood of $p$.

Proof of Theorem 2.a. By our assumptions it holds that $H_{r}^{\prime}(x) \geq H_{r}(x)$ for $x \in W$. This and (10) imply that

$$
\Phi_{r}\left(\Lambda\left(\mu^{\prime}\right)(x)\right)-\Phi_{r}(\Lambda(\mu)(x)) \geq 0, \quad x \in W .
$$

Since $M$ remains above $M^{\prime}$, we have $\vec{k}(p)-\overrightarrow{k^{\prime}}(p) \in \overline{\mathcal{O}^{n}}$. It follows from our assumptions and Lemma 3 that $\overrightarrow{k^{\prime}}(p) \in \overline{\Gamma_{r}}$. We claim that $\overrightarrow{k^{\prime}}(p) \in \partial \Gamma_{r}$. Otherwise, by Lemma 4.1 in Fontenele-Silva (2001), we would have that $\vec{k}(p) \in \Gamma_{r}$, which is a contradiction since $H_{r}(p) \leq 0$. So $\overrightarrow{k^{\prime}}(p) \in Z_{1}=\partial \Gamma_{r}$. We can use Lemma 4.1 in Fontenele-Silva (2001) to conclude that $\vec{k}(p) \in Z_{1}=\partial \Gamma_{r}$. As in the proof of Theorem 1.a, we can use Lemmas 1 and 2 to obtain that 
$\vec{k}(p)=\overrightarrow{k^{\prime}}(p)$. Since $\frac{\partial \sigma_{r}}{\partial x_{i}}>0$ on $\Gamma_{r}, \quad 1 \leq i \leq n, \vec{k}(p)=\overrightarrow{k^{\prime}}(p)$ is an elliptic root of $\sigma_{r}=0$ and $\vec{k}(p)=\overrightarrow{k^{\prime}}(p) \in \partial \Gamma_{r}$, we deduce that

$$
\frac{\partial \sigma_{r}}{\partial x_{i}}(\vec{k}(p))>0, \forall i=1, \ldots, n .
$$

Now, proceeding as in the proof of Theorem 1.a, we conclude that $\Phi_{r}$ is elliptic with respect to the functions $(1-t) \mu+t \mu^{\prime}, t \in[0,1]$. It follows from (17) and the maximum principle that $\mu$ and $\mu^{\prime}$ coincide in a neighborhood of zero. Therefore, $M$ and $M^{\prime}$ coincide in a neighborhood of $p$.

\section{ACKNOWLEDGMENTS}

We would like to thank M.P. do Carmo for suggesting us to publish this work.

\section{RESUMO}

Neste trabalho nós generalizamos e estendemos para uma variedade Riemanniana arbitrária princípios do máximo para hipersuperfícies com $r$-ésima curvatura média zero no espaço Euclidiano, obtidos por HounieLeite.

Palavras-chave: princípio do máximo, hipersuperfície, $r$-ésima curvatura média.

\section{REFERENCES}

Alexandrov AD. 1962. Uniqueness theorems for surfaces in the large I. Amer Math Soc Transl, Ser 2, 21: 341-354.

Caffarelli L, Nirenberg L and Spruck J. 1985. The Dirichlet problem for nonlinear second order elliptic equations III: Functions of the eigenvalues of the hessian. Acta Math 155: 261-301.

Fontenele F AND Silva SL. 2001. A tangency principle and applications. Illinois J Math 45: 213-228.

GÅRDING L. 1959. An inequality for hyperbolic polynomials. J Math Mech 8: 957-965.

Hounie J AND Leite ML. 1995. The maximum principle for hypersurfaces with vanishing curvature functions. J Differ Geom 41: 247-258.

Hounie J AND LeITe ML. 1999. Two-ended hypersurfaces with zero scalar curvature. Indiana Univ Math J 48: 817-882. 